\title{
Variational PDE models in image processing
}

\author{
Tony F. Chan, Jianhong (Jackie) Shen, and Luminita Vese *
}

July 31, 2002

\section{Contents}

1 Introduction $\mathbf{2}$

1.1 Image processing as an input-output system . . . . . . . . . 2

1.2 Image modeling and representation . . . . . . . . . . . 3

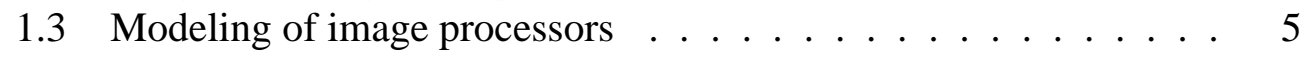

1.4 Variational PDE method ............... 6

2 Variational Image Inpainting and Interpolation 6

2.1 From Shannon's Theorem to variational inpainting . . . . . . . 7

2.2 Variational inpainting based on geometric image models . . . . . 9

3 Variational Level Set Image Segmentation 15

3.1 Active contours without edges and multi-phase level sets . . . . 16

3.2 Piecewise smooth Mumford-Shah segmentation . . . . . . . . 20

3.3 Logic operators for multi-channel image segmentation . . . . . . 24

* This paper is based on a plenary presentation given by Tony F. Chan at the 2002 Joint Mathematical Meeting, San Diego, and has been supported in part by NSF under grant numbers DMS-9973341 (Chan), DMS-0202565 (Shen), and ITR-0113439 (Vese), by ONR under N0001402-1-0015 (Chan), and by NIH under NIH-P20MH65166 (Chan and Vese). For the preprints and reprints mentioned in this paper, please visit our web site at: www.math.ucla.edu/ imagers. Chan and Vese are with the Department of Mathematics, UCLA, Los Angeles, CA 90095, \{chan, lvese\}@math.ucla.edu; Shen is with the School of Mathematics, University of Minnesota, Minneapolis, MN 55455, jhshen@ math.umn.edu. 


\section{Introduction}

Image processing, a traditionally engineering field, has attracted the attention of many mathematicians during the past two decades. From the vision and cognitive science point of view, image processing is a basic tool used to reconstruct the relative order, geometry, topology, patterns, and dynamics of the 3-D world from 2-D images. Therefore, it cannot be merely a historic coincidence that mathematics must meet image processing in this digital technology era.

The role of mathematics is also determined by the broad range of applications of image processing in contemporary science and technology. These include astronomy and aerospace exploration, medical imaging, molecular imaging, computer graphics, human and machine vision, telecommunication, auto-piloting, surveillance video, and biometric security identification (such as fingerprints and face identification), etc. All these highly diversified disciplines have made it necessary to develop the common mathematical foundation and frameworks for image analysis and processing. Mathematics at all levels must be introduced to meet the crucial qualities demanded by this new era - genericity, well-posedness, accuracy, and computational efficiency, just to name a few. In return, image processing has created tremendous opportunities for mathematical modeling, analysis, and computation.

In this article, we intend to give a broad picture of mathematical image processing through one of the most recent and very successful approaches - the variational PDE method. We first discuss two crucial ingredients for image processing: image modeling or representation, and processor modeling. We then focus on the variational PDE method. The backbone of the article consists of two major problems in image processing - inpainting and segmentation, which we have personally worked on, but by no means do we intend to have a comprehensive review of the entire field of image processing.

\subsection{Image processing as an input-output system}

Directly connected to image processing are the two dual fields in the contemporary computer science - computer vision and computer graphics. Vision (whether machine or human) is to reconstruct the 3-D world from the observed 2-D images, while graphics pursues the opposite direction in designing suitable 2-D scene images to simulate our 3-D world. Image processing is the crucial middle way connecting the two. 
Most abstractly, image processing can be considered as an input-output system

$$
Q_{0} \longrightarrow \text { Image Processor } \mathcal{T} \longrightarrow Q
$$

Here $\mathcal{T}$ denotes a typical image processor, for example, denoising, deblurring, segmentation, compression, or inpainting. The input data $Q_{0}$ can represent an observed or measured single image or image sequence, and the output $Q=$ $\left(q_{1}, q_{2}, \cdots\right)$ contains all the targeted image features.

For example, the human vision system can be considered as a highly involved multi-level image processor $\mathcal{T}$. $Q_{0}$ represents the image sequence that is constantly projected onto the retina. The output vector $Q$ contains all the major features that are important to our daily life, from the low-level ones such as relative orders, shapes, and grouping rules, to high-level feature parameters that help classify or identify various patterns and objects.

Listed in Table 1 are some typical image processing problems.

The two main ingredients of image processing are the input $Q_{0}$ and the processor $\mathcal{T}$. As a result, the two key issues that have been driving the entire mainstream mathematical research on image processing are (a) the modeling and representation of the input visual data $Q_{0}$, and (b) the modeling of the processing operators $\mathcal{T}$. The two are independent but also closely connected to each other by the universal rule in mathematics: the structure and performance of an operator $\mathcal{T}$ is greatly influenced by how the input class of functions are modeled or represented.

\subsection{Image modeling and representation}

To efficiently handle and process images, first we need to understand what images really are mathematically and how to represent them. For example, is it adequate to treat them as general $L^{2}$ functions, or a subset of $L^{2}$ with suitable regularity constraints? Among the various approaches, here we briefly outline three major classes of image modeling and representation.

Random fields modeling. An observed image $u_{0}$ is modeled as the sampling of a random field. For example, the Ising spin model in statistical mechanics can be used to model binary images. More generally, images are modeled by some Gibbs/Markovian random fields [29, 57]. The statistical properties of the fields are often established through the filtering technique and learning theory. Random field modeling is the most ideal approach for describing natural images with rich texture patterns such as trees and mountains.

Wavelets representation. Whether digitally or biologically, an image is often acquired from the responses of a collection of micro sensors (or photo receptors). 


\begin{tabular}{|l|l|l|}
\hline $\mathcal{T}$ & $Q_{0}$ & $Q$ \\
\hline denoising+deblurring & $u_{0}=K u+n$ & clean \& sharp $u$ \\
\hline inpainting & $\left.u_{0}\right|_{\Omega \backslash D}$ & entire image $\left.u\right|_{\Omega}$ \\
\hline segmenation & $u_{0}$ & $\begin{array}{l}\text { "objects" } \\
{\left[u_{k}, \Omega_{k}\right], k=1,2 \ldots}\end{array}$ \\
\hline scale-space & $u_{0}$ & $\begin{array}{l}\text { multiscale images } \\
\left(u_{\lambda_{1}}, u_{\lambda_{2}}, \ldots\right)\end{array}$ \\
\hline motion estimation & $\left(u_{0}^{(1)}, u_{0}^{(2)}, \ldots\right)$ & $\begin{array}{l}\text { optical flows } \\
\left(\vec{v}^{(1)}, \vec{v}^{(2)}, \ldots\right)\end{array}$ \\
\hline
\end{tabular}

Table 1: Typical image processors and their inputs and outputs. The symbols represent (1) $K$ : a blurring kernel, and $n$ : an additive noise, both assumed to be linear for simplicity in the current paper; (2) $u_{0}$ : the given noisy or blurred image; (3) $\Omega$ : the entire image domain, and $D$ : a subset where image information is missing or unaccessible; (4) $\left[u_{k}, \Omega_{k}\right]: \Omega_{k}$ 's are the segmented individual "objects," while $u_{k}$ 's are their intensity values; (5) $\lambda_{k}$ 's are different scales, and $u_{\lambda}$ can be roughly understood as the projection of the input image at scale $\lambda$; (6) $u_{0}^{(n)}$ 's denote the discrete sampling of a continuous "movie" $u_{0}(x, t)$ (with some small time step $h$ ), $\vec{v}^{(n)}$ 's are the estimated optical flows (i.e. velocity fields) at each moment.

During the past two decades, it has been gradually realized or experimentally supported that such local responses can be well approximated by wavelets. Wavelets as a new representation tool has revolutionized our notion of images and their multiscale structures [25, 33]. The new JPEG2000 protocol and the successful compression of the FBI fingerprints database are its two most influential applications. The theory is still being actively pushed forward by the new generation of geometric wavelets such as curvelets [7] and beamlets [42].

Regularity spaces. In the linear filtering theory of conventional digital image processing, an image $u$ is considered to be in the Sobolev space $H^{1}(\Omega)$. Sobolev model works well for homogeneous regions but is insufficient as a global image model, since it "smears" the most important visual cue - edges [34]. Two well known models have been introduced to legalize the existence of edges. One is 
Mumford and Shah's “object-edge" model [39], and the other is Rudin, Osher, and Fatemi's BV image model [43]. The object-edge model assumes that an ideal image $u$ consists of disjoint homogeneous object patches $\left[u_{k}, \Omega_{k}\right]$ with $u_{k} \in H^{1}\left(\Omega_{k}\right)$ and regular boundaries $\partial \Omega_{k}$ (characterized by the 1-dimensional Hausdorff measure). The BV image model assumes that an ideal image has bounded total variation $\int_{\Omega}|D u|$. All these regularity based image models are generally applicable to images with low texture patterns and without rapidly oscillatory components [36].

\subsection{Modeling of image processors}

How images are modeled and represented very much determines the way we model image processors. We shall illustrate this viewpoint through the example of denoising $u=\mathcal{T} u_{0}: u_{0}=u+n$, assuming for simplicity that the white noise $n$ is additive and homogeneous, and there is no blurring involved.

When images are represented by wavelets, the denoising processor $\mathcal{T}$ is in some sense "diagonalized," and equivalent to a simple engineering on the individual wavelet components. This is the celebrated results of Donoho and Johnstone on the thresholding based denoising schemes [27].

Under the statistical/random field modeling of images, the denoising processor $\mathcal{T}$ becomes the MAP (Maximum A Posteriori) estimation. By Bayes' formula, the posterior probability given an observation $u_{0}$ is

$$
p\left(u \mid u_{0}\right)=p\left(u_{0} \mid u\right) p(u) / p\left(u_{0}\right)
$$

The denoising processor $\mathcal{T}$ is achieved by solving the MAP problem $\max _{u} p\left(u \mid u_{0}\right)$. Therefore, besides the random field image model $p(u)$, it is also important to know the mechanism by which $u_{0}$ is generated from the ideal image $u$ (or the so called generative data model). The two are crucial for successfully carrying out Bayesian denoising.

Finally, if the ideal image $u$ is modeled as an element in certain regular function spaces such as $H^{1}(\Omega)$ or $\mathrm{BV}(\Omega)$, then the denoising processor $\mathcal{T}$ can be realized by a variational optimization. For instance, by Rudin-Osher-Fatemi's BV image model, $\mathcal{T}$ is achieved by

$$
\min _{u} \int_{\Omega}|D u| \quad \text { subject to } \frac{1}{|\Omega|} \int_{\Omega}\left(u-u_{0}\right)^{2} d x \leq \sigma^{2},
$$

where the white noise is assumed to be well approximated by Gaussian $N\left(0, \sigma^{2}\right)$. This is the well known denoising model first proposed by Rudin, Osher, and Fatemi, and belongs to the more general class of regularized data fitting models. 
Like using different coordinate systems to describe a single physical object, the different formulations of a same image processor are closely interconnected. Again take denoising for example. It has been shown that the wavelet technique is equivalent to an approximate optimal regularization in certain Besov spaces [23]. On the other hand, Bayesian processing and the regularity based variational approach can also be connected (at least formally) by the Gibbs' formula in statistical mechanics [30] (see the next section).

\subsection{Variational PDE method}

Having briefly introduced the general picture of mathematical image processing, we now focus on the variational PDE method through two processors: inpainting and segmentation.

For the history and a detailed description of the current developments of the variational and PDE method in image and vision analysis, we refer to the two special issues in IEEE Trans. Image Processing [7(3), 1998] and J. Visual Comm. Image Rep. [13(1/2), 2002], and also two recent monographs [2, 48].

In the variational or "energy" based models, nonlinear PDEs emerge as one derives their formal Euler-Lagrange equations, or tries to locate the local or global minima by the gradient descent method. Some of the PDEs can be studied by the viscosity solution approach [24], while many others still remain open to further theoretical investigation.

Compared with other approaches, the variational and PDE method has remarkable advantages in both theory and computation. First, it allows to directly handle and process visually important geometric features such as gradients, tangents, curvatures, and level sets. It can also effectively simulate several visually meaningful dynamic processes such as linear and nonlinear diffusions, and the information transport mechanism. Secondly, in terms of computation, it can profoundly benefit from the existing wealthy literature of numerical analysis and computational PDEs. For example, various well designed shock capturing schemes in Computational Fluid Dynamics (CFD) can be conveniently adapted to edge computation in images.

\section{Variational Image Inpainting and Interpolation}

The word inpainting is an artistic synonym for image interpolation, as initially circulated among museum restoration artists who manually restore cracked an- 
cient paintings. The concept of digital inpainting was first introduced into digital image processing in the paper by Bertalmio et al. [5]. Currently, digital inpainting techniques have found broad applications in image processing, vision analysis, and digital technologies, such as image restoration, disocclusion, perceptual image coding, zooming and image super-resolution, error concealment in wireless image transmission, and so on $[15,16,17,18]$. See for instance Figure 1 for the application in error concealment.

We now discuss the mathematical ideas and methodologies behind the variational inpainting techniques. Throughout this section, $u$ denotes the original complete image on a 2-D domain $\Omega$, and $u_{0}$ the observed or measured portion of $u$ on a subdomain or general subset $D$, which can be either noisy or blurry. The goal of inpainting is to recover $u$ on the entire image domain $\Omega$ as faithfully as possible from the available data $u_{0}$ on $D$.

\subsection{From Shannon's Theorem to variational inpainting}

Interpolation is a classical topic in approximation theory, numerical analysis, and signal and image processing. Successful interpolants include polynomials, harmonic waves, radially symmetric functions, finite elements, splines, wavelets, etc. Despite the diversity of the literature, there indeed exists one most widely recognized result due to Shannon [50], known as Shannon's Sampling Theorem.

Theorem 1 (Shannon's Theorem) If a signal $u(t)$ is bandlimited within $(-\omega, \omega)$, then,

$$
u(t)=\sum_{n=-\infty}^{\infty} u\left(n \frac{\pi}{\omega}\right) \operatorname{sinc}\left(\frac{\omega}{\pi} t-n\right)
$$

That is, if an analog signal $u(t)$ (with finite energy, or equivalently, in $L^{2}(\mathbb{R})$ ) does not contain any high frequencies, then it can be perfectly interpolated from its properly sampled discrete sequence $u_{0}[n]=u(n \pi / \omega)$ (with $\omega / \pi$ known as the Nyquist frequency).

All interpolation problems share this "if-then" structure. "If" specifies the space where the target signal $u$ is to be looked for, while "then" gives the reconstruction or interpolation procedure based on the discrete samples (or more generally, any partial information about the signal).

Unfortunately, for most real applications in signal and image processing, one cannot expect a closed-form formula as clean as Shannon's. This is at least due 
to two factors. First, signals like images are intrinsically not bandlimited because of the presence of edges (or Heaviside type singularities) for vision analysis and communication [34]. Secondly, for most real applications, the given incomplete data are often noisy and even blurred during the imaging or transimission processes. Therefore, in the criterion of Shannon's Theorem, we are dealing with a class of "bad" signals $u$ with "unreliable" samples $u_{0}$.

Naturally, for image inpainting, both the "if" and "then" statements in Shannon's Theorem need to be modeled carefully. It turns out that there are two powerful and interdependent frameworks that can carry out this task: one is the variational method, and the other, the Bayesian framework [29].

In the Bayesian approach, the "if"-statement specifies both the so-called prior model and the data model. The prior model specifies how a priori images are distributed, or equivalently, which images occur more frequently than the others. Probabilistically, it specifies the prior probability $p(u)$. Let $u_{0}$ denote the incomplete data that are observed, measured, or sampled. Then the second part of "if" is to model how $u_{0}$ is generated from $u: u \rightarrow u_{0}$, or to specify the conditional probability $p\left(u_{0} \mid u\right)$. Finally, in the Bayesian framework, Shannon's "then"-statement is replaced by the Maximum A Posteriori (MAP) optimization:

$$
\max _{u} p\left(u \mid u_{0}\right)=p\left(u_{0} \mid u\right) p(u) / p\left(u_{0}\right)
$$

where we have spelled out Bayes' formula. (It is also equivalent to maximizing the product of the prior model and data model, since the denominator is a fixed normalization constant once $u_{0}$ is given.) To summarize, Bayesian inpainting is to find the most probable image given its incomplete and possibly distorted observation.

The variational approach resembles the Bayesian methodology, only now everything is expressed deterministically. The Bayesian prior model $p(u)$ becomes the specification of the regularity of an image $u$, while the data model $p\left(u_{0} \mid u\right)$ now measures how well the observation $u_{0}$ is fitted if the original image is indeed $u$. Regularity is enforced through "energy" functionals, for example, the Sobolev norm $E[u]=\int_{\Omega}|\nabla u|^{2} d x$, the total variation model of Rudin, Osher, and Fatemi $[31,43] E[u]=\int_{\Omega}|D u|$, and the Mumford-Shah free-boundary model [39] $E[u, \Gamma]=\int_{\Omega \backslash \Gamma}|\nabla u|^{2} d x+\beta H^{1}(\Gamma)$, with $H^{1}$ denoting the 1-D Hausdorff measure. The quality of data fitting $u \rightarrow u_{0}$ is often judged by an error 
measure $E\left[u_{0} \mid u\right]$. For instance, the least square measure [51] prevails in the literature due to the genericity of Gaussian type noises and the Central Limit Theorem: $E\left[u_{0} \mid u\right]=\frac{1}{|D|} \int_{D}\left(T u-u_{0}\right)^{2} d x$, where $D$ is the domain on which $u_{0}$ has been sampled or measured, $|D|$ its area or cardinality for the discrete case, and $T$ denotes any linear or nonlinear image processor (such as blurring and diffusion). In this variational setting, Shannon's "then"-statement becomes a constrained optimization problem:

$$
\min E[u] \quad \text { over all } u: \quad E\left[u_{0} \mid u\right] \leq \sigma^{2} .
$$

Here $\sigma^{2}$ denotes the variance of the white noise, which is assumed to be known by proper statistical estimators. Equivalently, the model solves the following unconstrained problem using Lagrange multiplier: $\lambda[9]$,

$$
\min _{u} E[u]+\lambda E\left[u_{0} \mid u\right] .
$$

Generally, $\lambda$ expresses the balance between regularity and fitting. In summary, variational inpainting is to search for the most "regular" image that best fits the given observation.

The Bayesian approach is more universal in the sense of allowing general statistical prior and data models, and is powerful for restoring both artificial images and natural images (or textures). But the learning of the prior model and the data model is usually quite expensive. The variational approach is ideal for dealing with regularity and geometry, but tends to work best for man-made indoor and outdoor scenes, or images with low textures. The two approaches (1) and (2) can be at least formally unified under Gibbs' formula in statistical mechanics [30]:

$$
E[\cdot] \propto-\beta \log p(\cdot), \quad \text { or } \quad p(\cdot) \propto e^{-E[\cdot] / \beta},
$$

where $\beta=k T$ is the product of the Boltzmann constant and temperature, and $\propto$ means equality up to a multiplicative or additive constant. (However, the definability of a rigorous probability measure over "all" images is highly non-trivial because of the multi-scale nature of images. Recent efforts can be found in Mumford and Gidas [38].)

\subsection{Variational inpainting based on geometric image models}

In a typical image inpainting problem, $u_{0}$ denotes the observed or measured incomplete portion of a clean "good" image $u$ on the entire image domain $\Omega$. A 
simplified but already very powerful data model in various digital applications is blurring followed by noise degradation and spatial restriction:

$$
\left.u_{0}\right|_{D}=(K u+n)_{D}
$$

where $K$ is a continuous blurring kernel, often assumed to be linear or even shiftinvariant, and $n$, an additive white noise field assumed to be close to Gaussian for simplicity. $\left.u_{0}\right|_{\Omega \backslash D}$ is missing or inaccessible. The goal of inpainting is to reconstruct $u$ as faithfully as possible from $\left.u_{0}\right|_{D}$. The data model is explicitly given by

$$
E\left[u_{0} \mid u, D\right]=\frac{1}{|D|} \int_{D}\left(K u-u_{0}\right)^{2} d x
$$

Therefore, from the variational point of view, the quality of an inpainting model crucially depends on the prior model or the regularity energy $E[u]$.

The TV prior model $E[u]=\int_{\Omega}|D u|$ was first introduced into image processing by Rudin, Osher, Fatemi in [43]. Unlike the Sobolev image model $E_{2}[u]=$ $\int_{\Omega}|\nabla u|^{2} d x$, the TV model legalizes one of the most important vision features the "edges." For example, for a cartoon image $u$ showing the night sky $(u=0)$ with a full bright moon $(u=1)$, the Sobolev energy blows up, while the TV energy $\int_{\Omega}|D u|=$ the perimeter of the moon, which is finite. Therefore, in combination with the data model (4), the variational TV inpainting model is to minimize

$$
E_{\mathrm{tv}}\left[u \mid u_{0}, D\right]=\alpha \int_{\Omega}|D u|+\lambda \int_{D}\left(K u-u_{0}\right)^{2} d x
$$

The admissible space is $\mathrm{BV}(\Omega)$, the Banach space of all functions with bounded variation [31]. We observe that it is very similar to the celebrated TV restoration model of Rudin, Osher, and Fatemi [43]. In fact, the beauty and power exactly lie in that the model provides a unified framework for denoising, deblurring, and image reconstruction from incomplete data. Figure 1 displays the computational output of the model as applied to the error concealment of a blurry image with simulated random packet loss due to the transmission failure of a network.

The second well-known prior model is Mumford-Shah's object-edge model [39]. Unlike TV, the edge set $\Gamma$ is now explicitly singled out, and an image $u$ is understood as the combination of both the geometric feature $\Gamma$ and the piecewise smooth 

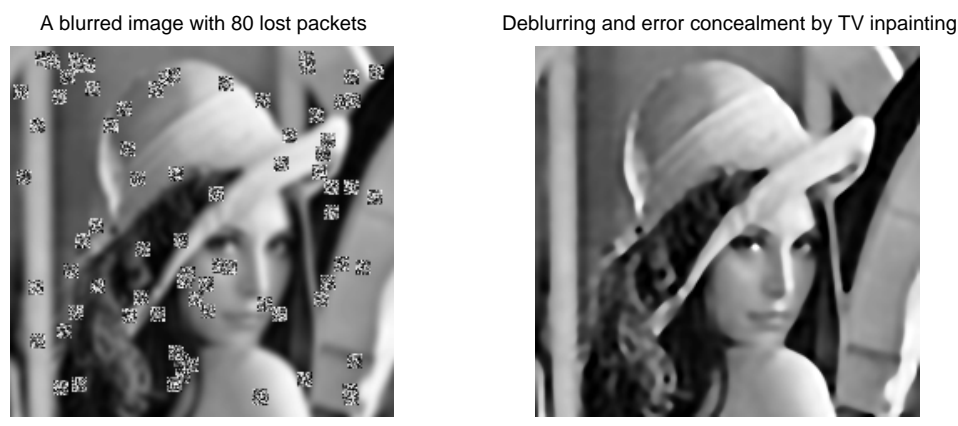

Figure 1: TV inpainting for the error concealment of a blurry image.

"objects" $u_{i}$ 's on all the connected components $\Omega_{i}$ 's of $\Omega \backslash \Gamma$. Thus in both the Bayesian and variational languages, the prior model consists of two parts (see (3)):

$$
\begin{aligned}
& p(u, \Gamma)=p(u \mid \Gamma) p(\Gamma) \quad \text { and } \\
& E[u, \Gamma]=E[u \mid \Gamma]+E[\Gamma] .
\end{aligned}
$$

In the Mumford-Shah model, the edge regularity is specified by $E[\Gamma]=H^{1}(\Gamma)$, the 1-D Hausdorff measure, or as in most computational applications, $E[\Gamma]=$ length $(\Gamma)$ assuming that $\Gamma$ is Lipschitz. The smoothness of the "objects" is naturally characterized by the ordinary Sobolev norm: $E[u \mid \Gamma]=\int_{\Omega \backslash \Gamma}|\nabla u|^{2} d x$. Therefore, in combination with the data model (4), the variational inpainting model based on the Mumford-Shah prior is given by

$$
\begin{aligned}
& \inf _{u, \Gamma} E_{\mathrm{ms}}\left[u, \Gamma \mid u_{0}, D\right]= \\
& \alpha \int_{\Omega \backslash \Gamma}|\nabla u|^{2} d x+\beta H^{1}(\Gamma)+\lambda \int_{D}\left(K u-u_{0}\right)^{2} d x .
\end{aligned}
$$

Figure 2 shows one application of this model for text removal [28]. Note edges are preserved and smooth regions remain smooth.

Numerous applications have demonstrated that, for classical applications in denoising, deblurring or segmentation, both the TV and Mumford-Shah models perform sufficiently well even by the high standard of human vision. But inpainting does have its special identity. We have demonstrated in $[15,28]$ that for large-scale inpainting problems, high order image models which incorporate the curvature information become necessary for more faithful visual effects. 

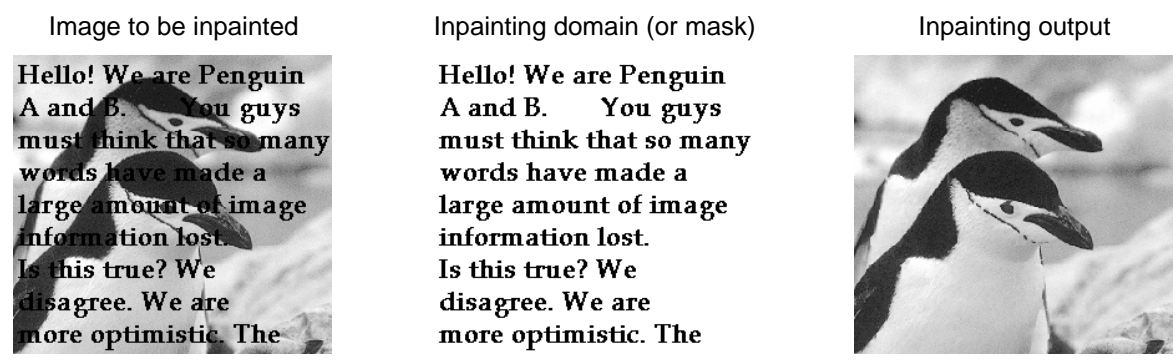

Figure 2: Mumford-Shah inpainting for text removal.

The key to high order geometric image models is Euler's elastica curve model:

$$
e[\gamma]=\int_{\gamma}\left(a+b \kappa^{2}\right) d s, \quad a, b>0,
$$

where $\kappa$ denotes the scalar curvature. Birkhoff and de Boor [6] called it the "nonlinear spline" model in approximation theory. It was first introduced into computer vision by Mumford [37]. Unlike straight lines (for which $b=0$ ), the elastica model allows smooth curves because of the curvature term, which is important for computer vision and computer graphics.

By imposing the elastica energy on each individual level lines of $u$ (at least symbolically or by assuming that $u$ is regular enough), we obtain the so-called elastica image model:

$$
\begin{aligned}
E_{\mathrm{el}[}[u] & =\int_{-\infty}^{\infty} e[u \equiv \lambda] d \lambda \\
& =\int_{-\infty}^{\infty} \int_{u \equiv \lambda}\left(a+b \kappa^{2}\right) d s d \lambda \\
& =\int_{\Omega}\left(a+b \kappa^{2}\right)|\nabla u| d x .
\end{aligned}
$$

In the last integrand, the curvature is given by $\kappa=\nabla \cdot[\nabla u /|\nabla u|]$. (Notice that in the absence of the curvature term, the above formula is exactly the co-area formula for smooth functions [31].) This elastica prior model was first studied for inpainting by Masnou and Morel [35], and Chan, Kang, and Shen [11], and it improves the TV inpainting model as expected.

Similarly, the Mumford-Shah image model $E_{\mathrm{ms}}$ can also be improved by hav- 
ing the length energy replaced by Euler's elastica energy:

$$
E_{\mathrm{mse}}[u, \Gamma]=\alpha \int_{\Omega \backslash \Gamma}|\nabla u|^{2} d x+e[\Gamma] .
$$

It was first applied to image inpainting by Esedoglu and Shen [28]. Figure 3 shows one example of applying this image prior model to the inpainting of an occluded disk. Both the TV and Mumford-Shah inpainting models would complete the interpolation with a straight line edge and introduce visible corners a result. The elastica model restores the smooth boundary.
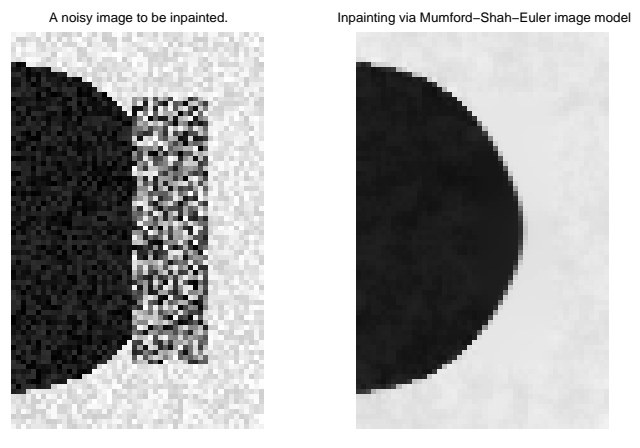

Figure 3: Smooth inpainting by the Mumford-Shah-Euler model.

The improved performance of curvature based models comes at a price, both in terms of theory and computation. The existence and uniqueness of the TV and Mumford-Shah inpainting models can be studied in a fashion similar to the classical restoration and segmentation problems. But theoretical study on the high order models is only in the very beginning. The difficulty lies in the involvement of the second order geometric feature - curvature, and the identification of a proper function space to study the models. Secondly in terms of computation, the calculus of variation on the curvature term leads to fourth-order highly nonlinear PDEs, whose fast and efficient numerical solution imposes a tremendous challenge.

Let us conclude this section with a brief discussion on computation, especially for the TV and Mumford-Shah inpaintings.

For the TV inpainting model $E_{\mathrm{tv}}$, the Euler-Lagrange equation is formally (or assuming that $u$ is in the Sobolev space $W^{1,1}$ ) given by

$$
-\nabla \cdot\left[\frac{\nabla u}{|\nabla u|}\right]+\mu K^{*} \chi_{D}\left(K u-u_{0}\right)=0 .
$$


Here $K^{*}$ denotes the adjoint of the linear blurring kernel $K$, the multiplier $\chi_{D}(x)$ the indicator of $D$, and $\mu=2 \lambda / \alpha$. The boundary condition along $\partial \Omega$ is Neumann adiabatic, to eliminate any boundary contribution during the integration-by-parts process. This nonlinear PDE can be solved iteratively by the freezing technique: let $u^{(n)}$ denote the current inpainting at step $n$, then the updated inpainting $u^{(n+1)}$ solves the linearized PDE

$$
-\nabla \cdot\left[\frac{\nabla u^{(n+1)}}{\left|\nabla u^{(n)}\right|}\right]+\mu K^{*} \chi_{D}\left(K u^{(n+1)}-u_{0}\right)=0 .
$$

In practice, the intermediate diffusivity coefficient $1 /\left|\nabla u^{(n)}\right|$ is often modified to $1 / \sqrt{\left|\nabla u^{(n)}\right|^{2}+\epsilon^{2}}$ for some small conditioning parameter $\epsilon$, or by the mandatory ceiling and flooring between $\epsilon$ and $1 / \epsilon$. The convergence of such algorithms have been well studied in the literature $[9,26]$. There are also many other techniques possible for solving (8) in the literature, for example, see Vogel and Oman [54], and Chan, Mulet, and Golub [10]. We only need to relate (8) to the conventional TV restoration case.

The computation of the Mumford-Shah inpainting model is also very interesting. Unlike segmentation, for inpainting, one's direct interest is only in $u$, not $\Gamma$. Such understanding makes the $\Gamma$-convergence approximation theory perfect for inpainting. According to Ambrosio and Tortorelli [1], by introducing an edge signature function $z(x) \in[0,1], x \in \Omega$, and having $E[u \mid \Gamma]=\alpha \int_{\Omega \backslash \Gamma}|\nabla u|^{2} d x$ replaced by $E[u \mid z]=\alpha \int_{\Omega} z^{2}|\nabla u|^{2} d x$, one can approximate the length energy in the Mumford-Shah model by a quadratic integral in $z$ (up to a constant multiplier):

$$
E_{\epsilon}[z]=\beta \int_{\Omega}\left(\frac{\epsilon|\nabla z|^{2}}{2}+\frac{(z-1)^{2}}{2 \epsilon}\right) d x, \quad \epsilon \ll 1 .
$$

Thus the Mumford-Shah inpainting model is approximated by

$$
E_{\epsilon}\left[u, z \mid u_{0}, D\right]=E[u \mid z]+E_{\epsilon}[z]+\lambda E\left[u_{0} \mid u, D\right],
$$

which is a quadratic integral in both $u$ and $z$ ! It leads to a coupled system of linear elliptic type of PDE's in both $u$ and the edge signature $z$, which can be solved efficiently using any numerical elliptic solver. The example in Figure 2 has been computed by this scheme [28].

Finally we should also mention some of the major applications of the inpainting and geometric image interpolation models developed above. These include 
digital zooming, primal-sketch based perceptual image coding, error concealment for wireless image transmission, and progressive disocclusion in computer vision [15]. Extensions to color or more general hyperspectral images, and nonflat image features (i.e., that live on Riemannian manifolds [14]) are also currently being studied in the literature. Other approaches to the inpainting problem can be found in [3, 5, 8]. In particular, it has been just recently pointed out in [4] that the PDE inpainting model in [5] is closely related to the stream function-vorticity equation in fluid dynamics.

\section{Variational Level Set Image Segmentation}

Images are the proper 2-D projections of the 3-D world containing various objects. To successfully reconstruct the 3-D world, or at least approximately, the first crucial step is to identify the regions in images that correspond to individual objects. This is the well known problem of image segmentation. It has broad applications in a variety of important fields such as computer vision and medical image processing.

Denote by $u_{0}$ an observed image on a 2-D Lipschitz open and bounded domain $\Omega$. Segmentation is to find a visually meaningful edge set $\Gamma$ which leads to a complete partition of $\Omega$. Each connected component $\Omega_{i}$ of $\Omega \backslash \Gamma$ should correspond to at most one real physical object or pattern in our 3-D world, for example, the white matter in brain images or the abnormal tissues in organs. In some applications, one is also interested in the clean image patches $u_{i}$ on each $\Omega_{i}$ of the segmentation, since $u_{0}$ is often noisy.

Therefore, there are two crucial ingredients in the mathematical modeling and computation of the segmentation problem. The first is how to formulate a model that appropriately combines the effects of both the edge set $\Gamma$ and its segmented regions $\left\{\Omega_{i}, i=1,2, \cdots\right\}$. And the other is to find the most efficient way to represent the geometry of both the edge set and the regions, and to represent the segmentation model as a result. This of course reflects the general philosophy in the introduction section.

In the variational PDE approach, these two issues have found good answers in the literature. For the first, one observes the celebrated segmentation model of Mumford and Shah [39], and the second, the level set representation technology of Osher and Sethian [40]. In what follows, we detail our recent efforts in advancing the application of the level set technology to various Mumford-Shah related image segmentation models. Much of the works can be found in our papers [19, 20, 22, 
$21,53]$, and also in the related works [52, 55, 41, 44, 58].

We start with a novel active contour model whose formulation is independent of intensity edges defined by the gradients, in contrast to most conventional ones in the literature. We then explain how this model can be efficiently computed based on the multi-phase level set method. In the second part, we extend these results to the level set formulation and computation of the general Mumford-Shah segmentation model for piecewise smooth images. In the last part, we present our recent work on extending the previous models to the logical operations on multi-channel image objects.

\subsection{Active contours without edges and multi-phase level sets}

Active contour is a powerful tool in image and vision analysis for boundary detection and object segmentation. The key idea is to evolve a curve so that it eventually stops along the object edges of the given image $u_{0}$. The curve evolution is controlled by two sorts of energies: the internal energy which defines the regularity of the curve, and the external one determined by the given image $u_{0}$. The latter is often called the feature-driven energy.

In almost all classical active contour models, the feature-driven energies rely heavily on the gradient feature $\left|\nabla u_{0}\right|$ or its smoothed version $\left|\nabla G_{\sigma} * u_{0}\right|$, where $G_{\sigma}$ denotes a Gaussian kernel with a small variance $\sigma$. They work well for detecting gradient-defined edges, but fail for more general classes of edges, such as the boundary of a nebula in some astronomic images or the top image in Figure 4.

Our new model - active contours without edges, first introduced in [19, 20], is formulated independent of the gradient information, and therefore can handle more general types of edges. The model is to minimize the energy

$$
E_{2}\left[c_{1}, c_{2}, \Gamma \mid u_{0}\right]=\int_{\operatorname{int}(\Gamma)}\left|u_{0}(x)-c_{1}\right|^{2} d x+\int_{\operatorname{ext}(\Gamma)}\left|u_{0}(x)-c_{2}\right|^{2} d x+\nu|\Gamma|,
$$

where $\nu>0$, and $\operatorname{int}(\Gamma)$ and $\operatorname{ext}(\Gamma)$ denote the interior and exterior of $\Gamma$, and $|\Gamma|$ its length. The subscript 2 in $E_{2}$ indicates that it deals with two-phase images, i.e., ones whose "objects" can be completely indexed by the interior and exterior of $\Gamma$.

In the level set formulation of Osher and Sethian [40], $\Gamma$ is embedded as the zero level set $\{\phi=0\}$ of a Lipschitz continuous function $\phi: \Omega \rightarrow \mathbb{R}$. Consequently, $\{\phi>0\}$ and $\{\phi<0\}$ define the interior $\Omega^{+}$and exterior $\Omega^{-}$of the curve. (Computationally, the level set approach is superior to other curve representations in both letting one directly work on a fixed rectangular grid and allowing automatic topological changes such as merging and breaking.) Denote by $H$ the 
1-dimensional Heaviside function: $H(z)=1$ if $z \geq 0$ and 0 if $z<0$. Then the energy in our model becomes

$$
\begin{aligned}
E_{2}\left[c_{1}, c_{2}, \phi \mid u_{0}\right] & =\int_{\Omega}\left|u_{0}(x)-c_{1}\right|^{2} H(\phi) d x+\int_{\Omega}\left|u_{0}(x)-c_{2}\right|^{2}(1-H(\phi)) d x \\
& +\nu \int_{\Omega}|\nabla H(\phi)| d x
\end{aligned}
$$

Minimizing $E_{2}\left[c_{1}, c_{2}, \phi \mid u_{0}\right]$ with respect to $c_{1}, c_{2}$ and $\phi$ leads to the Euler-Lagrange equation:

$$
\begin{aligned}
\frac{\partial \phi}{\partial t} & =\delta(\phi)\left[\nu \operatorname{div}\left(\frac{\nabla \phi}{|\nabla \phi|}\right)-\left|u_{0}-c_{1}\right|^{2}+\left|u_{0}-c_{2}\right|^{2}\right] \\
c_{1}(t) & =\frac{\int_{\Omega} u_{0}(x) H(\phi(x)) d x}{\int_{\Omega} H(\phi(x)) d x}, \quad c_{2}(t)=\frac{\int_{\Omega} u_{0}(x)(1-H(\phi(x))) d x}{\int_{\Omega}(1-H(\phi(x))) d x},
\end{aligned}
$$

with a suitable initial guess $\phi(0, x)=\phi_{0}(x)$. In numerical implementations, the Heaviside function $H(z)$ is often regularized by some $H_{\varepsilon}(z)$ in $C^{1}(\mathbb{R})$, so that as $\varepsilon \rightarrow 0$, the latter converges to $H(z)$ in some suitable sense. As a result, the Dirac function $\delta(z)$ in the last equation is regularized to $\delta_{\varepsilon}(z)=H_{\varepsilon}^{\prime}(z)$. We have discovered in [20] that a carefully designed approximation scheme can even allow interior contours to emerge, which has been a challenging task for most conventional algorithms. Also notice that the length term in the energy has led to the mean curvature motion.

The model performs as an active contour in the class of piecewise constant images taking only two values, looking for a two-phase segmentation of a given image. The internal energy is defined by the length, while the external energy is independent of the gradient $\left|\nabla u_{0}\right|$. Defining the segmented image by $u(x)=$ $c_{1} H(\phi(x))+c_{2}(1-H(\phi(x)))$, we realize that the energy model is exactly the Mumford-Shah segmentation model [39] restricted to the class of piecewise constant images. However, our model was initially developed from the active contour point of view.

Two typical numerical outputs of the model are displayed in Figure 4. The top row shows that our model can segment and detect objects without clear gradient edges. The bottom one shows that it can also capture complicated boundaries and interior contours.

For more complicated situations where multiple objects occlude each other and multi-phase edges such as T-junctions emerge, the above two-phase active contour model becomes insufficient and we need to introduce more than one level 


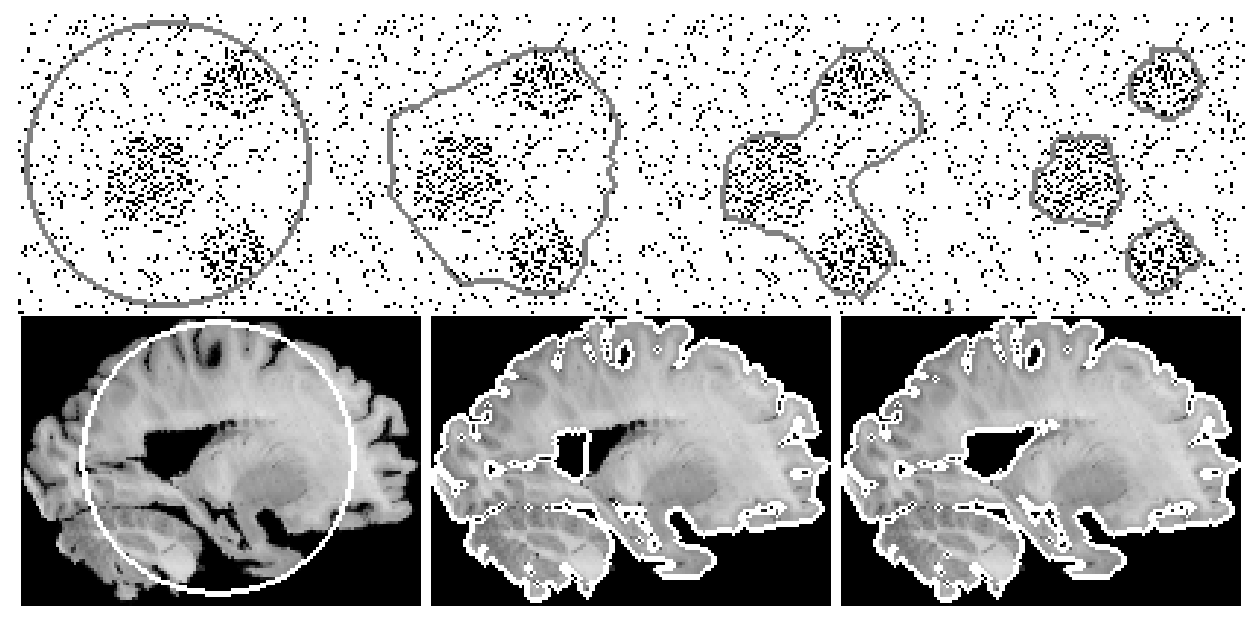

Figure 4: Top: detection of a simulated minefield by our new active contour model. Bottom: segmentation of an MRI brain image. Notice that the interior boundaries are automatically detected.

set functions. In $[22,53]$, we generalize the above framework to multi-phase active contours, or equivalently, the piecewise constant Mumford-Shah segmentation with multi-phase regions:

$$
\inf _{u, \Gamma} E_{\mathrm{ms}}\left[u, \Gamma \mid u_{0}\right]=\sum_{i} \int_{\Omega_{i}}\left|u_{0}-c_{i}\right|^{2} d x+\nu|\Gamma| .
$$

Here, $\Omega_{i}$ 's denote the connected components of $\Omega \backslash \Gamma$, and $u=c_{i}$ on $\Omega_{i}$. Notice that $\Gamma$ can now be a general set of edge curves, including for example the $\mathrm{T}$ junction class.

Generally, consider $m$ level set functions $\phi_{i}: \Omega \rightarrow \mathbb{R}$. The union of the zerolevel sets of $\phi_{i}$ represents the edges in the segmented image. Using these $m$ level set functions, one can define up to $n=2^{m}$ phases, which form a disjoint and complete partitioning of $\Omega$. Therefore, each point $x \in \Omega$ belongs to one and only one phase. In particular, there is no vacuum or overlap among the phases. This is an important advantage, compared with the classical multi-phase representation in $[44,56]$, where a level set function is associated to each phase, and therefore more level set functions are needed. Figure 5 shows two typical examples of multi-phase partitioning corresponding to $m=2$ and 3 .

We now illustrate the multi-phase level set approach through the example of $n=4$ and $m=2$. Let $c=\left(c_{11}, c_{10}, c_{01}, c_{00}\right)$ denote a constant vector, and 


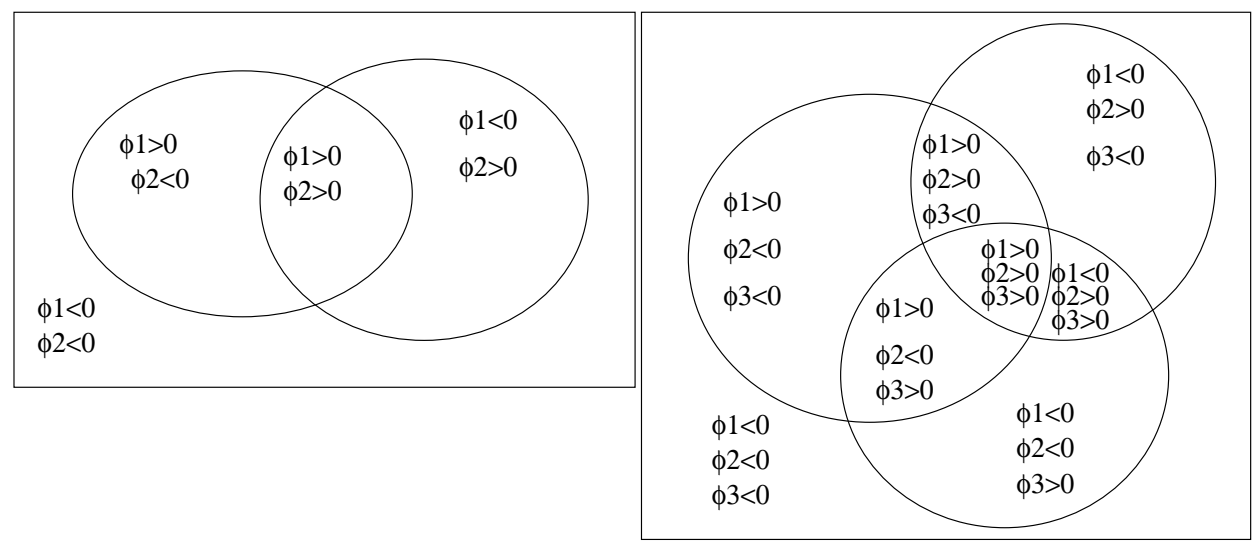

Figure 5: Left: two curves given by $\phi_{1}=0$ and $\phi_{2}=0$, partition the domain into four regions based on indicator vector $\left(\operatorname{sign}\left(\phi_{1}\right), \operatorname{sign}\left(\phi_{2}\right)\right)$. Right: Three curves given by $\phi_{1}=0, \phi_{2}=0$, and $\phi_{3}=0$, partition the domain into eight regions based on the triple $\left(\operatorname{sign}\left(\phi_{1}\right), \operatorname{sign}\left(\phi_{2}\right), \operatorname{sign}\left(\phi_{3}\right)\right)$.

$\Phi=\left(\phi_{1}, \phi_{2}\right)$ the two-phase level set vector. Then we are looking for an ideal image $u$ in the form of

$$
\begin{array}{r}
u=c_{11} H\left(\phi_{1}\right) H\left(\phi_{2}\right)+c_{10} H\left(\phi_{1}\right)\left(1-H\left(\phi_{2}\right)\right) \\
+c_{01}\left(1-H\left(\phi_{1}\right)\right) H\left(\phi_{2}\right)+c_{00}\left(1-H\left(\phi_{1}\right)\right)\left(1-H\left(\phi_{2}\right)\right) .
\end{array}
$$

The Mumford-Shah segmentation energy becomes

$$
\begin{array}{r}
E_{4}\left[c, \Phi \mid u_{0}\right]=\int_{\Omega}\left|u_{0}(x)-c_{11}\right|^{2} H\left(\phi_{1}\right) H\left(\phi_{2}\right) d x \\
+\int_{\Omega}\left|u_{0}(x)-c_{10}\right|^{2} H\left(\phi_{1}\right)\left(1-H\left(\phi_{2}\right)\right) d x \\
\quad+\int_{\Omega}\left|u_{0}(x)-c_{01}\right|^{2}\left(1-H\left(\phi_{1}\right)\right) H\left(\phi_{2}\right) d x \\
+\int_{\Omega}\left|u_{0}(x)-c_{00}\right|^{2}\left(1-H\left(\phi_{1}\right)\right)\left(1-H\left(\phi_{2}\right)\right) d x \\
\quad+\nu \int_{\Omega}\left|\nabla H\left(\phi_{1}\right)\right| d x+\nu \int_{\Omega}\left|\nabla H\left(\phi_{2}\right)\right| d x .
\end{array}
$$

Its minimization leads to the Euler-Lagrange equations. First, with $\Phi$ fixed, the $c$ minimizer can be explicitly worked out as before:

$$
c_{i j}(t)=\text { average of } u_{0} \text { on }\left\{(2 i-1) \phi_{1}>0,(2 j-1) \phi_{2}>0\right\} \quad i, j=0,1 .
$$


In return, this new $c$ information leads to the Euler-Lagrange equations for $\Phi$ :

$$
\begin{array}{r}
\frac{\partial \phi_{1}}{\partial t}=\delta\left(\phi_{1}\right)\left[\nu \operatorname{div}\left(\frac{\nabla \phi_{1}}{\left|\nabla \phi_{1}\right|}\right)-\left(\left(u_{0}-c_{11}\right)^{2}-\left(u_{0}-c_{01}\right)^{2}\right) H\left(\phi_{2}\right)\right. \\
\left.-\left(\left(u_{0}-c_{10}\right)^{2}-\left(u_{0}-c_{00}\right)^{2}\right)\left(1-H\left(\phi_{2}\right)\right)\right], \\
\begin{aligned}
\frac{\partial \phi_{2}}{\partial t}=\delta\left(\phi_{2}\right)\left[\nu \operatorname{div}\left(\frac{\nabla \phi_{2}}{\left|\nabla \phi_{2}\right|}\right)-\left(\left(u_{0}-c_{11}\right)^{2}-\left(u_{0}-c_{01}\right)^{2}\right) H\left(\phi_{1}\right)\right. \\
\left.-\left(\left(u_{0}-c_{10}\right)^{2}-\left(u_{0}-c_{00}\right)^{2}\right)\left(1-H\left(\phi_{1}\right)\right)\right] .
\end{aligned}
\end{array}
$$

Note that the equations are governed by both the mean curvatures and jumps of the data energy terms across the boundary.

Figure 6 shows an application of the model to the medical analysis of a brain image. Displayed are the final segmented image and its associated four phases. Our model successfully identifies and segments the white and gray matters.

Recently the above models and algorithms have been extended to multi-channel, volumetric, and texture images in $[12,13,46]$. Let us give a little more details about texture segmentation from [46]. Texture images refer to general images of natural scenes, such as grasslands, beaches, rocks, mountains, and human body tissues. They typically carry certain coherent structures in scales, orientations, and local frequencies. To segment texture images using the above models, we first apply Gabor's filters to extract these coherent structures. The filter responses create a new vectorial (or multi-channel) feature image in the form of $U(x)=\left(u_{\alpha}(x), u_{\beta}(x), \cdots, u_{\gamma}(x)\right)$, where the Greek letters stand for the filter signatures and typically each takes a value of (scale, orientation, local frequency). We then apply the vectorial active-contour-without-edges model to the segmentation of $U$. Figure 7 shows one typical example.

\subsection{Piecewise smooth Mumford-Shah segmentation}

The most general Mumford-Shah piecewise smooth segmentation [39] is defined by

$$
\inf _{u, \Gamma} E_{\mathrm{ms}}\left[u, \Gamma \mid u_{0}\right]=\int_{\Omega}\left|u-u_{0}\right|^{2} d x+\mu \int_{\Omega \backslash \Gamma}|\nabla u|^{2} d x+\nu|\Gamma|,
$$

where $\mu, \nu$ are positive parameters. It allows the segmented "objects" to have smoothly varying intensities, instead of being strictly constant. We now show 


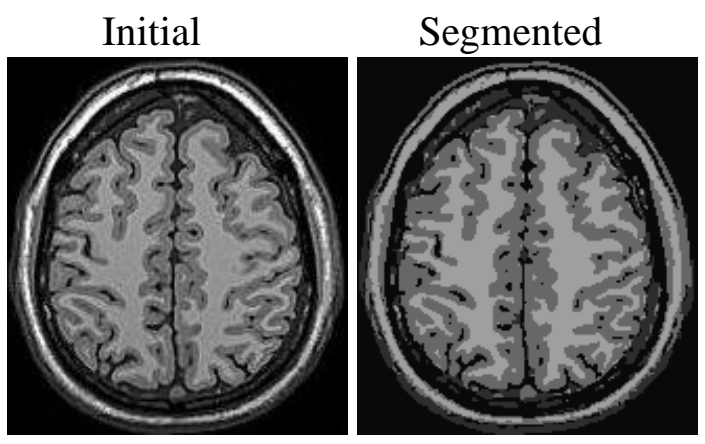

Final four segments

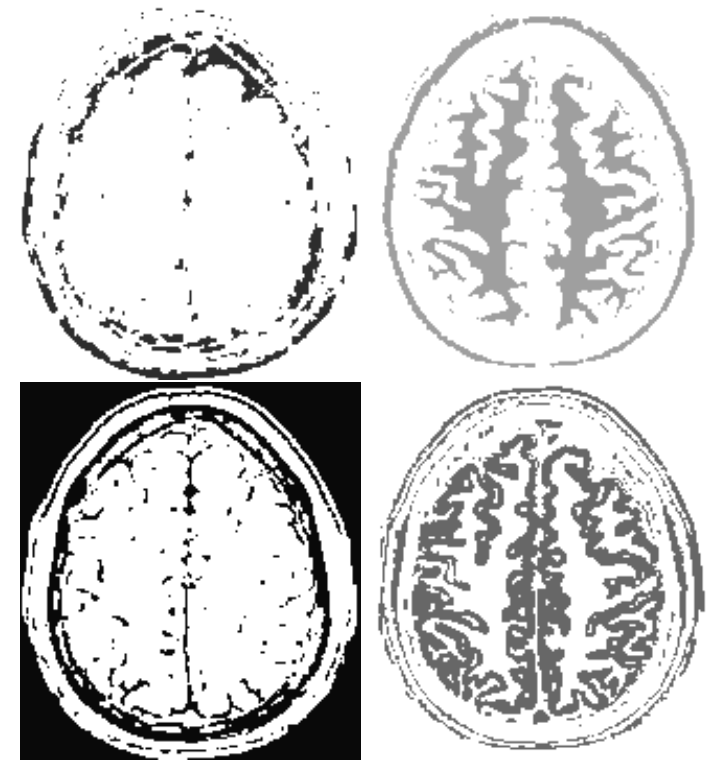

Figure 6: The original and segmented images (top row), and the final four segments (the rest). 

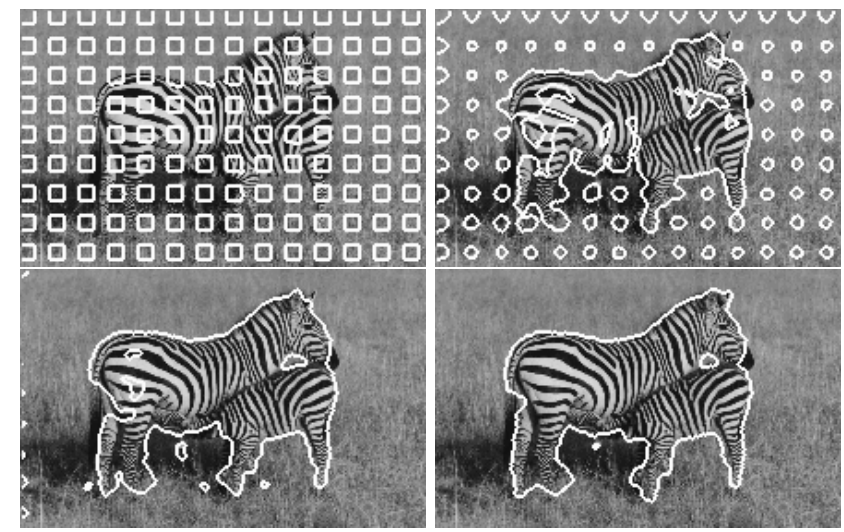

Figure 7: An example of texture segmentation (at increasing times).

how to carry out the model based on the multi-phase level set approach [20]. As before, we start with the two-phase situation where a single level set function $\phi$ is sufficient, followed by the more general multi-phase case.

In the two-phase situation, the ideal image $u$ is segmented to $u^{ \pm}$by the level set function $\phi$ :

$$
u(x)=u^{+}(x) H(\phi(x))+u^{-}(x)(1-H(\phi(x))) .
$$

We assume that both $u^{+}$and $u^{-}$are $C^{1}$ functions up to the boundary $\{\phi=0\}$. Substituting this expression into (12), we obtain

$$
\begin{aligned}
& E\left[u^{+}, u^{-}, \phi \mid u_{0}\right]=\int_{\Omega}\left|u^{+}-u_{0}\right|^{2} H(\phi) d x+\int_{\Omega}\left|u^{-}-u_{0}\right|^{2}(1-H(\phi)) d x \\
& \left.+\mu \int_{\Omega}\left|\nabla u^{+}\right|^{2} H(\phi)\right) d x+\mu \int_{\Omega}\left|\nabla u^{-}\right|^{2}(1-H(\phi)) d x+\nu \int_{\Omega}|\nabla H(\phi)| .
\end{aligned}
$$

First with $\phi$ fixed, the variation on $E\left[u^{+}, u^{-}, \phi \mid u_{0}\right]$ leads to the two EulerLagrange equations for $u^{ \pm}$separately:

$$
u^{ \pm}-u_{0}=\mu \triangle u^{ \pm} \text {on } \pm \phi>0, \quad \frac{\partial u^{ \pm}}{\partial \vec{n}}=0 \text { on }\{\phi=0\} .
$$

(Here \pm takes either + or - , but uniformly across the formula.) They act as the denoising operators on the homogeneous regions only. Notice that no smoothing is done across the boundary $\{\phi=0\}$, which is very important in image analysis. 
Next, keeping the functions $u^{+}$and $u^{-}$fixed, and minimizing $E\left[u^{+}, u^{-}, \phi \mid u_{0}\right]$ with respect to $\phi$, we obtain the motion of the zero level set:

$$
\frac{\partial \phi}{\partial t}=\delta(\phi)\left[\nu \nabla\left(\frac{\nabla \phi}{|\nabla \phi|}\right)-\left(\left|u^{+}-u_{0}\right|^{2}+\mu\left|\nabla u^{+}\right|^{2}-\left|u^{-}-u_{0}\right|^{2}-\mu\left|\nabla u^{-}\right|^{2}\right)\right],
$$

with some initial guess $\phi(t=0, x)$. The above equation is actually computed at least near a narrow band of the zero level set. As a result, computationally, we have to continuously extend both $u^{+}$and $u^{-}$from their original domains $\{ \pm \phi>0\}$ to a suitable neighborhood of the zero level set $\{\phi=0\}$. Figure 8 displays an application of the model in astronomical image analysis. Although the nebula itself does not seem to be a smooth object, the piesewise smooth model can still correctly capture all the main features.
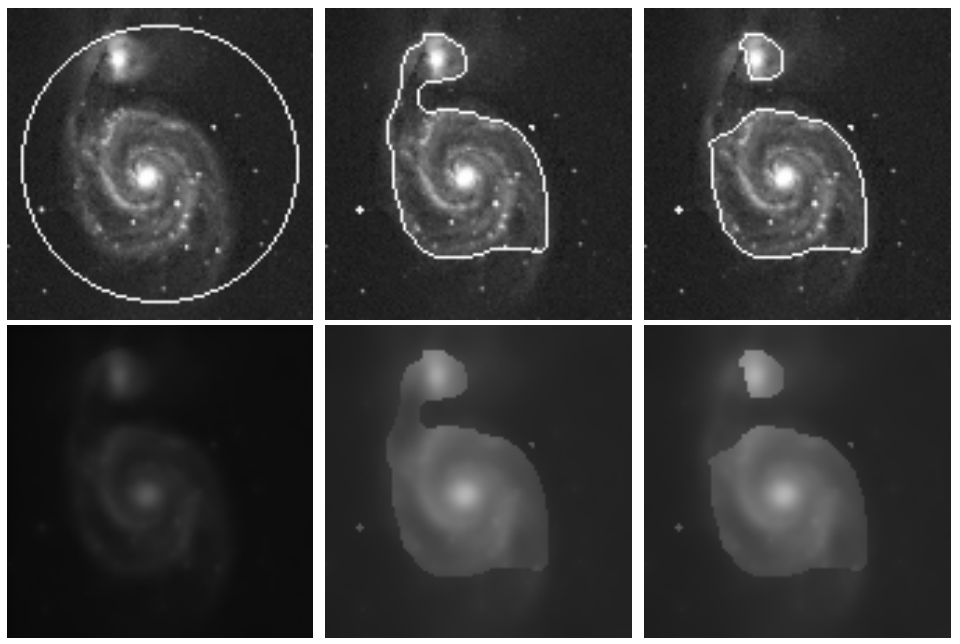

Figure 8: Numerical result from the piecewise smooth Mumford-Shah level set algorithm with one level set function.

As in the previous section, there are cases where the boundaries forming a complete partition of the image cannot be represented by a single level set function. Then one has to turn to the multi-phase approach. In our papers, thanks to the planer Four Color Theorem, we have been able to conclude that two level set functions are sufficient for any multi-phase partition problems.

By the Four Color Theorem, one can color all the regions in a partition using only four colors, so that any two adjacent regions are color distinguishable. Iden- 
tifying a phase with one color, we see that two level set functions $\phi_{1}$ and $\phi_{2}$ are sufficient to produce four "colors:" $\left\{ \pm \phi_{1}>0, \pm \phi_{2}>0\right\}$. Therefore, they can completely segment a general image with a multi-phase boundary set $\Gamma$ given by $\left\{\phi_{1}=0\right\}$ or $\left\{\phi_{2}=0\right\}$. As before, we do not have the problems of "overlapping" or "vacuum" as in $[56,44]$. Note that by this formulation, generally each "color" can still have many isolated components. Therefore, the segmentation is complete only after one applies an extra step of the well known topological processor for finding the connected components of an open set.

In this four-phase formulation, the ideal image $u$ is segmented into four disjoint but complete parts $u^{ \pm \pm}$, each defined by one of the four phases:

$$
\left\{ \pm \phi_{1}>0, \pm \phi_{2}>0\right\}
$$

Overall, by using the Heaviside function, we obtain the following synthesis formula:

$$
\begin{array}{r}
u=u^{++} H\left(\phi_{1}\right) H\left(\phi_{2}\right)+u^{+-} H\left(\phi_{1}\right)\left(1-H\left(\phi_{2}\right)\right) \\
+u^{-+}\left(1-H\left(\phi_{1}\right)\right) H\left(\phi_{2}\right)+u^{--}\left(1-H\left(\phi_{1}\right)\right)\left(1-H\left(\phi_{2}\right)\right),
\end{array}
$$

for all $x \in \Omega$. We can express in a similar way the energy function of $u$ and $\Phi=\left(\phi_{1}, \phi_{2}\right)$, and derive the corresponding Euler-Lagrange equations.

Notice the remarkable feature of this single model, which includes both the original energy formulation and the elliptic and evolutionary PDEs: it naturally combines all the three image processors - active contour, segmentation, and denoising.

\subsection{Logic operators for multi-channel image segmentation}

In a multi-channel image $u(x)=\left(u_{1}(x), u_{2}(x), \cdots, u_{n}(x)\right)$, a single physical object can leave different traces in different channels. For example, Figure 9 shows a two-channel image containing a triangle which is however incomplete in each individual channel. For this example, most conventional segmentation models for multi-channel images $[12,13,32,47,49,58]$ would output the complete triangle, i.e., the union of both channels. The union is just one of the several possible logical operations for multi-channel images. For example, the intersection and the differentiation are also very common in applications, as illustrated in Figure 10.

In this section, we outline our recent efforts in developing logical segmentation schemes for multi-channel images based on the active-contour-without-edges model [45]. 


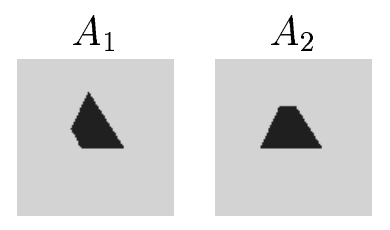

Figure 9: A synthetic example of an object in two different channels. Notice that the lower left corner of $A_{1}$ and the upper corner of $A_{2}$ are missing.

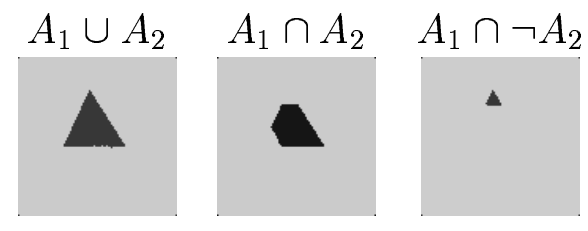

Figure 10: Different logical combinations for the sample image: the union, the intersection, and the differentiation.

First, we define two logical variables to encode the information inside and outside the contour $\Gamma$ separately for each channel $i$ :

$$
\begin{aligned}
& z_{i}^{\text {in }}\left(u_{0}^{i}, x, \Gamma\right)= \begin{cases}1 & \text { if } x \text { is inside } \Gamma \text { and not on the object } \\
0 & \text { otherwise }\end{cases} \\
& z_{i}^{\text {out }}\left(u_{0}^{i}, x, \Gamma\right)= \begin{cases}1 & \text { if } x \text { is outside } \Gamma \text { and on the object, } \\
0 & \text { otherwise. }\end{cases}
\end{aligned}
$$

Such different treatments are motivated by the energy minimization formulation. Intuitively speaking, in order for the active coutour $\Gamma$ to evolve and eventually capture the exact boundary of the targeted logical object, the energy should be designed so that both partial capture and over capture lead to high energies (corresponding to $z_{i}^{\text {out }}=1$ and $z_{i}^{i n}=1$ separately). Imagine that the target object is the tumor tissue, then in terms of decision theory, over and partial captures correspond to false alarms and misses separately. Both are to be penalized.

In practice, we do not have the precise information of "the object," which is exactly to be segmented. One possible way to approximate $z_{i}^{i n}$ and $z_{i}^{\text {out }}$ is based 


\begin{tabular}{|l||l|l|l|l|r|r|r|}
\hline \multicolumn{7}{|c|}{ Truth table for the two-channel case } \\
\hline & $z_{1}^{\text {in }}$ & $z_{2}^{\text {in }}$ & $z_{1}^{\text {out }}$ & $z_{2}^{\text {out }}$ & $A_{1} \cup A_{2}$ & $A_{1} \cap A_{2}$ & $A_{1} \cap \neg A_{2}$ \\
\hline \multirow{2}{*}{$\begin{array}{l}x \text { inside } \Gamma \\
\left(\text { or } x \in \Omega^{+}\right)\end{array}$} & 1 & 1 & 0 & 0 & 1 & 1 & 1 \\
\cline { 2 - 9 } & 1 & 0 & 0 & 0 & 0 & 1 & 1 \\
\cline { 2 - 8 } & 0 & 1 & 0 & 0 & 0 & 1 & 0 \\
\cline { 2 - 8 } & 0 & 0 & 0 & 0 & 0 & 0 & 1 \\
\hline \multirow{3}{*}{$\begin{array}{l}x \text { outside } \Gamma \\
\left.\text { (or } x \in \Omega^{-}\right)\end{array}$} & 0 & 0 & 1 & 1 & 1 & 1 & 0 \\
\cline { 2 - 8 } & 0 & 0 & 1 & 0 & 1 & 0 & 1 \\
\cline { 2 - 8 } & 0 & 0 & 0 & 1 & 1 & 0 & 0 \\
\cline { 2 - 8 } & 0 & 0 & 0 & 0 & 0 & 0 & 0 \\
\hline
\end{tabular}

Table 2: The truth table for two channels. Notice that inside $\Gamma$ "true" is represented by 0 . It is so designed to encourage the contour to enclose the targeted logical object at a lower energy cost.

on the interior $\left(\Omega^{+}\right)$and exterior $\left(\Omega^{-}\right)$averages $c_{i}^{ \pm}$in channel $i$ :

$$
\begin{aligned}
z_{i}^{i n}\left(u_{0}^{i}, x, \Gamma\right) & =\frac{\left|u_{0}^{i}(x)-c_{i}^{+}\right|^{2}}{\max _{y \in \Omega^{+}}\left|u_{0}^{i}(y)-c_{i}^{+}\right|^{2}}, \quad \text { for } x \in \Omega^{+} ; \\
z_{i}^{\text {out }}\left(u_{0}^{i}, x, \Gamma\right) & =\frac{\left|u_{0}^{i}(x)-c_{i}^{-}\right|^{2}}{\max _{y \in \Omega^{-}}\left|u_{0}^{i}(y)-c_{i}^{-}\right|^{2}}, \quad \text { for } x \in \Omega^{-} .
\end{aligned}
$$

The desired truth table can then be described using the $z_{i}^{\text {in }}$ 's and $z_{i}^{\text {out }}$ 's. In Table 2, we have shown three examples of logical operations for the two-channel case. Notice that "true" is represented by 0 inside $\Gamma$. It is so designed to encourage energy minimization when the contour tries to capture the targeted object inside.

We then design continuous objective functions to smoothly interpolate the binary truth table. This is because in practice, as mentioned above, the $z$ 's are approximated and take continuous values. For example, the possible interpolants for the union and intersection can be:

$$
\begin{aligned}
& f_{A_{1} \cup A_{2}}(x)=\sqrt{z_{1}^{\text {in }}(x) z_{2}^{\text {in }}(x)}+\left(1-\sqrt{\left(1-z_{1}^{\text {out }}(x)\right)\left(1-z_{2}^{\text {out }}(x)\right)}\right) \\
& f_{A_{1} \cap A_{2}}(x)=1-\sqrt{\left(1-z_{1}^{\text {in }}(x)\right)\left(1-z_{2}^{\text {in }}(x)\right)}+\sqrt{z_{1}^{\text {out }}(x) z_{2}^{\text {out }}(x)} .
\end{aligned}
$$

The square roots are taken to keep them of the same order as the original scalar models. It is straightforward to extend the two-channel case to more general $n$ channel ones. 
The energy functional $E$ for the logical objective function $f$ can be expressed by the level set function $\phi$. Generally, as just shown above, the objective function can be separated into two parts,

$$
f=f\left(z_{1}^{\text {in }}, z_{1}^{\text {out }}, \cdots, z_{n}^{\text {in }}, z_{n}^{\text {out }}\right)=f_{\text {in }}\left(z_{1}^{\text {in }}, \cdots, z_{n}^{\text {in }}\right)+f_{\text {out }}\left(z_{1}^{\text {out }}, \cdots, z_{n}^{\text {out }}\right) .
$$

The energy functional is then defined by

$$
\begin{aligned}
E\left[\phi \mid c^{+}, c^{-}\right] & =\mu \operatorname{length}(\phi=0)+\lambda \int_{\Omega}\left[f_{\text {in }}\left(z_{1}^{\text {in }}, \cdots, z_{n}^{\text {in }}\right) H(\phi)\right. \\
& \left.+f_{\text {out }}\left(z_{1}^{\text {out }}, \cdots, z_{n}^{\text {out }}\right)(1-H(\phi))\right] d x
\end{aligned}
$$

Here each $c^{ \pm}=\left(c_{1}^{ \pm}, \cdots, c_{n}^{ \pm}\right)$is in fact a multi-channel vector. The associated Euler-Lagrange equation is similar to the scalar model:

$$
\frac{\partial \phi}{\partial t}=\delta(\phi)\left[\mu \operatorname{div}\left(\frac{\nabla \phi}{|\nabla \phi|}\right)-\lambda\left(f_{\text {in }}\left(z_{1}^{\text {in }}, \cdots, z_{n}^{\text {in }}\right)-f_{\text {out }}\left(z_{1}^{\text {out }}, \cdots, z_{n}^{\text {out }}\right)\right)\right],
$$

with suitable boundary conditions as before. Even though the form often looks complicated for a typical application, its implementation is very similar to that of the scalar model.

Numerical results support our above efforts. Figure 9 shows two different occlusions of a triangle. We are able to successfully recover the union, the intersection, and the differentiation of the objects in Figure 10 using our model. In Figure 11, we have a two-channel image of the brain. In one we have a "tumor" with some noise, while the other is clear. The images are not registered. We want to find $A_{1} \cap \neg A_{2}$ so that the tumor can be observed. This happens to be a very complicated example as there are a lot of features and textures. However the model finds the tumor successfully.

\section{Acknowledgments}

Jackie Shen would like to thank his former advisors Professors Gilbert Strang and Stan Osher for their constant support and inspiration.

\section{References}

[1] L. Ambrosio and V. M. Tortorelli. Approximation of functionals depending on jumps by elliptic functionals via $\Gamma$-convergence. Comm. Pure Appl. Math., 43:999-1036, 1990. 


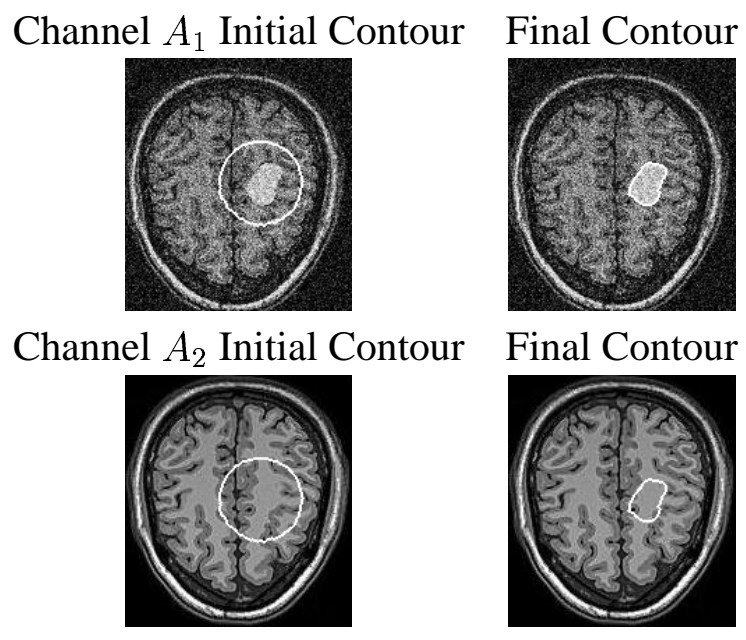

Figure 11: Region based logical model on a medical image. In the first channel $A_{1}$, the noisy image has a "brain tumor", while channel $A_{2}$ does not. The goal is to spot the tumor that is in channel $A_{1}$, but not in $A_{2}$, i.e., the differentiation $A_{1} \cap \neg A_{2}$. In the right column, we observe that the tumor has been successfully captured.

[2] G. Aubert and P. Kornprobst. Mathematical Problems in Image Processing: Partial Differential Equations and the Calculus of Variations, volume 147 of Applied Mathematical Sciences. Springer-Verlag, 2001.

[3] C. Ballester, M. Bertalmio, V. Caselles, G. Sapiro, and J. Verdera. Filling-in by joint interpolation of vector fields and grey levels. IEEE Trans. Image Process., 10(8):1200-1211, 2001.

[4] M. Bertalmio, A. L. Bertozzi, and G. Sapiro. Navier-Stokes, fluid dynamics, and image and video inpainting. IMA Preprint 1772 at: www.ima.umn.edu/preprints/jun01, June, 2001.

[5] M. Bertalmio, G. Sapiro, V. Caselles, and C. Ballester. Image inpainting. Computer Graphics, SIGGRAPH 2000, July, 2000.

[6] G. Birkhoff and C. R. De Boor. Piecewise polynomial interpolation and approximation. In H. Garabedian, editor, Approximation of Functions, pages 164-190. Elsevier, 1965. 
[7] E. J. Candés and D. L. Donoho. Curvelets and reconstruction of images from noisy radon data. Wavelet Applications in Signal and Image Processing VIII, A. Aldroubi, A. F. Laine, M. A. Unser, editors, Proc. SPIE 4119, 2000.

[8] V. Caselles, J.-M. Morel, and C. Sbert. An axiomatic approach to image interpolation. IEEE Trans. Image Processing, 7(3):376-386, 1998.

[9] A. Chambolle and P. L. Lions. Image recovery via total variational minimization and related problems. Numer. Math., 76:167-188, 1997.

[10] T. F. Chan, G. H. Golub, and P. Mulet. A nonlinear primal-dual method for total variation-based image restoration. SIAM J. Sci. Comput., 20(6):19641977, 1999.

[11] T. F. Chan, S.-H. Kang, and J. Shen. Euler's elastica and curvature based inpaintings. SIAM J. Appl. Math., in press, 2002.

[12] T. F. Chan, B. Sandberg, and L. Vese. Active contours without edges for vector-valued images. J. Visual Comm. Image Rep., 11:130-141, 1999.

[13] T. F. Chan, B. Sandberg, and L. Vese. Active contours without edges for textured images. UCLA Department of Mathematics CAM report 02-28, 2002 .

[14] T. F. Chan and J. Shen. Variational restoration of non-flat image features: models and algorithms. SIAM J. Appl. Math., 61(4):1338-1361, 2000.

[15] T. F. Chan and J. Shen. Mathematical models for local nontexture inpaintings. SIAM J. Appl. Math., 62(3):1019-1043, 2001.

[16] T. F. Chan and J. Shen. Morphologically invariant PDE inpaintings. UCLA Department of Mathematics CAM Report 01-15 at: www.math.ucla.edu/imagers; submitted to IEEE Trans. Image Process., 2001.

[17] T. F. Chan and J. Shen. Nontexture inpainting by curvature driven diffusions (CDD). J. Visual Comm. Image Rep., 12(4):436-449, 2001.

[18] T. F. Chan and J. Shen. On the role of the BV image model in image restoration. UCLA Department of Mathematics CAM Report 02-14 available at: www.math.ucla.edu//imagers. To appear in AMS Contemporary Mathematics, 2002. 
[19] T. F. Chan and L. A. Vese. An active contour model without edges. Lec. Notes Comput. Sci., 1682:141-151, 1999.

[20] T. F. Chan and L. A. Vese. Active contours without edges. IEEE Trans. Image Process., 10/2:266-277, 2001.

[21] T. F. Chan and L. A. Vese. A level set algorithm for minimizing the Mumford-Shah functional in image processing. IEEE/Computer Society Proceedings of the 1st IEEE Workshop on "Variational and Level Set Methods in Computer Vision”, pages 161-168, 2001.

[22] T. F. Chan and L. A. Vese. Active contour and segmentation models using geometric PDE's for medical imaging. Malladi, R. (Ed.), "Geometric Methods in Bio-Medical Image Processing”, Series: Mathematics and Visualization, Springer, 2002.

[23] A. Cohen, W. Dahmen, I. Daubechies, and R. DeVore. Harmonic analysis of the space BV. Acta Mathematica, submitted, 2000.

[24] M. G. Crandall, H. Ishii, and P. L. Lions. User's guide to viscosity solutions of second order partial linear differential equations. Bull. Amer. Math. Soc., 27:1-67, 1992.

[25] I. Daubechies. Ten Lectures on Wavelets. SIAM, Philadelphia, 1992.

[26] D. C. Dobson and C. R. Vogel. Convergence of an iterative method for total variation denoising. SIAM J. Numer. Anal., 34(5):1779-1791, 1997.

[27] D. L. Donoho and I. M. Johnstone. Ideal spacial adaption by wavelet shrinkage. Biometrika, 81:425-455, 1994.

[28] S. Esedoglu and J. Shen. Digital inpainting based on the Mumford-ShahEuler image model. European J. Appl. Math., in press, 2002.

[29] S. Geman and D. Geman. Stochastic relaxation, Gibbs distributions, and the Bayesian restoration of images. IEEE Trans. Pattern Anal. Machine Intell., 6:721-741, 1984.

[30] W. Gibbs. Elementary Principles of Statistical Mechanics. Yale University Press, 1902. 
[31] E. Giusti. Minimal Surfaces and Functions of Bounded Variation. Birkhäuser, Boston, 1984.

[32] F. Guichard. A morphological affine and Galilean invariant scale space for movies. IEEE Trans. Image Process., 7(3):444-456, 1998.

[33] S. Mallat. A Wavelet Tour of Signal Processing. Academic Press, 1998.

[34] D. Marr and E. Hildreth. Theory of edge detection. Proc. Royal Soc. London, B:207: 187-217, 1980.

[35] S. Masnou and J.-M. Morel. Level-lines based disocclusion. Proceedings of 5th IEEE Int'l Conf. on Image Process., Chicago, 3:259-263, 1998.

[36] Y. Meyer. Oscillating Patterns in Image Processing and Nonlinear Evolution Equations, volume 22 of University Lecture Series. AMS, Providence, 2001.

[37] D. Mumford. Elastica and computer vision. In C. L. Bajaj, editor, Algebraic Geometry and its Applications, pages 491-506. Springer-Verlag, New York, 1994.

[38] D. Mumford and B. Gidas. Stochastic models for generic images. Quaterly Applied Math., 59:85-111, 2001.

[39] D. Mumford and J. Shah. Optimal approximations by piecewise smooth functions and associated variational problems. Comm. Pure Applied. Math., 42:577-685, 1989.

[40] S. Osher and J. Sethian. Fronts propagating with curvature-dependent speed: Algorithms based on Hamilton-Jacobi formulation. J. Comput. Phys., 79:12-49, 1988.

[41] N. Paragios and R. Deriche. Coupled geodesic active regions for image segmentation: A level set approach. Proceedings of the 6th European Conference on Computer Vision, Dublin, Ireland, II:224-240, 2000.

[42] E. L. Pennec and S. Mallat. Image compression with geometrical wavelets. Proc. of Int'l Conf. Image Process., 1:661-664, 2000.

[43] L. Rudin, S. Osher, and E. Fatemi. Nonlinear total variation based noise removal algorithms. Physica D, 60:259-268, 1992. 
[44] C. Samson, L. Blanc-Féraud, G. Aubert, and J. Zerubia. A level set model for image classification. Int'l J. Computer Vision, 40/3:187-197, 2000.

[45] B. Sandberg and T. F. Chan. Logic operations for active contours on multichannel images. UCLA Department of Mathematics CAM Report 02-12, 2002 .

[46] B. Sandberg, T. F. Chan, and L. Vese. A level-set and Gabor-based active contour algorithm for segmenting textured images. UCLA Department of Mathematics CAM report 02-39, 2002.

[47] G. Sapiro. Color snakes. Computer Vision and Image Understanding, pages 247-253, 1997.

[48] G. Sapiro. Geometric Partial Differential Equations and Image Processing. Cambridge University Press, 2001.

[49] G. Sapiro and D. L. Ringach. Anisotropic diffusion of multi-valued images with applications to color filtering. IEEE Trans. Image Process., 5:15821586, 1996.

[50] C. E. Shannon. Communications in the presence of noise. Proc. I.R.E., $37: 10-21,1949$.

[51] G. Strang. Introduction to Applied Mathematics. Wellesley-Cambridge Press, MA, 1993.

[52] A. Tsai, A. Yezzi, and A.S. Willsky. Curve evolution implementation of the Mumford-Shah functional for image segmentation, denoising, interpolation, and magnification. IEEE Trans. Image Process., 10/8:1169-1186, 2001.

[53] L. A. Vese and T. F. Chan. A multiphase level set framework for image segmentation using the Mumford and Shah model. UCLA Department of Mathematics CAM Report 01-25, to appear in Int'l J. Computer Vision, 2001.

[54] C. R. Vogel and M. E. Oman. Iterative methods for total variation denoising. SIAM J. Sci. Comput., 17(1):227-238, 1996.

[55] A. Yezzi, A. Tsai, and A. Willsky. A statistical approach to snakes for bimodal and trimodal imagery. Proceedings of the 7th Int'l Conf. on Computer Vision, pages 898-903, 1999. 
[56] H.-K. Zhao, T. F. Chan, B. Merriman, and S. Osher. A variational level set approach to multiphase motion. J. Comput. Phys., 127, 179-195.

[57] S. C. Zhu, Y. N. Wu, and D. Mumford. Minimax entropy principle and its applications to texture modeling. Neural Computation, 9:1627-1660, 1997.

[58] S. C. Zhu and A. Yuille. Region competition: unifying snakes, region growing, and Bayes/MDL for multi-band image segmentation. IEEE Trans. Pattern Anal. Machine Intell., 18(9):884-900, 1996. 